\title{
Excess of Electron-Like Events in MiniBooNE
}

\author{
En-Chuan Huang \\ on behalf of MiniBooNE collaboration
}

Thisdocumentwas prepared by [MiniBooNECollaboration] using theresources oftheFermi National Accelerator Laboratory(Fermilab), aU.S. Department of Energy, Office ofScience, HEP UserFaaility. Fermilabis managed byFermi Research Alliance, $U C($ FRA), actingunderContractNo. DE-ACO2-07CH11359

Neutrino 2018, Heidelberg, Germany 


\section{Outline}

- The LSND Anomaly

- MiniBooNE experiment

- Observed excess

- Allowed region and other possibilities 


\section{LSND Anomaly}

\section{$800 \mathrm{MeV}$ proton beam}

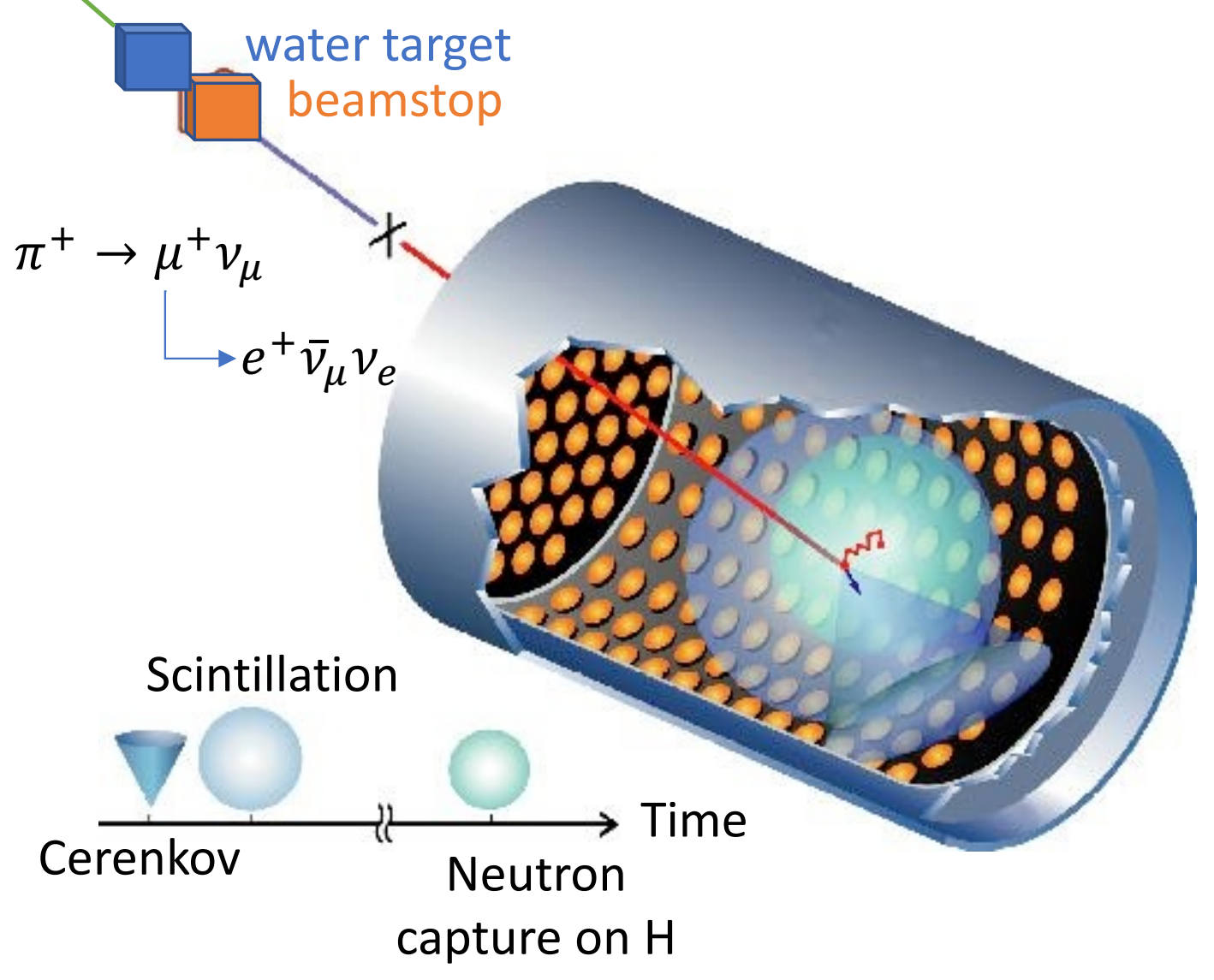

LSND observed a $3.8 \sigma$ excess
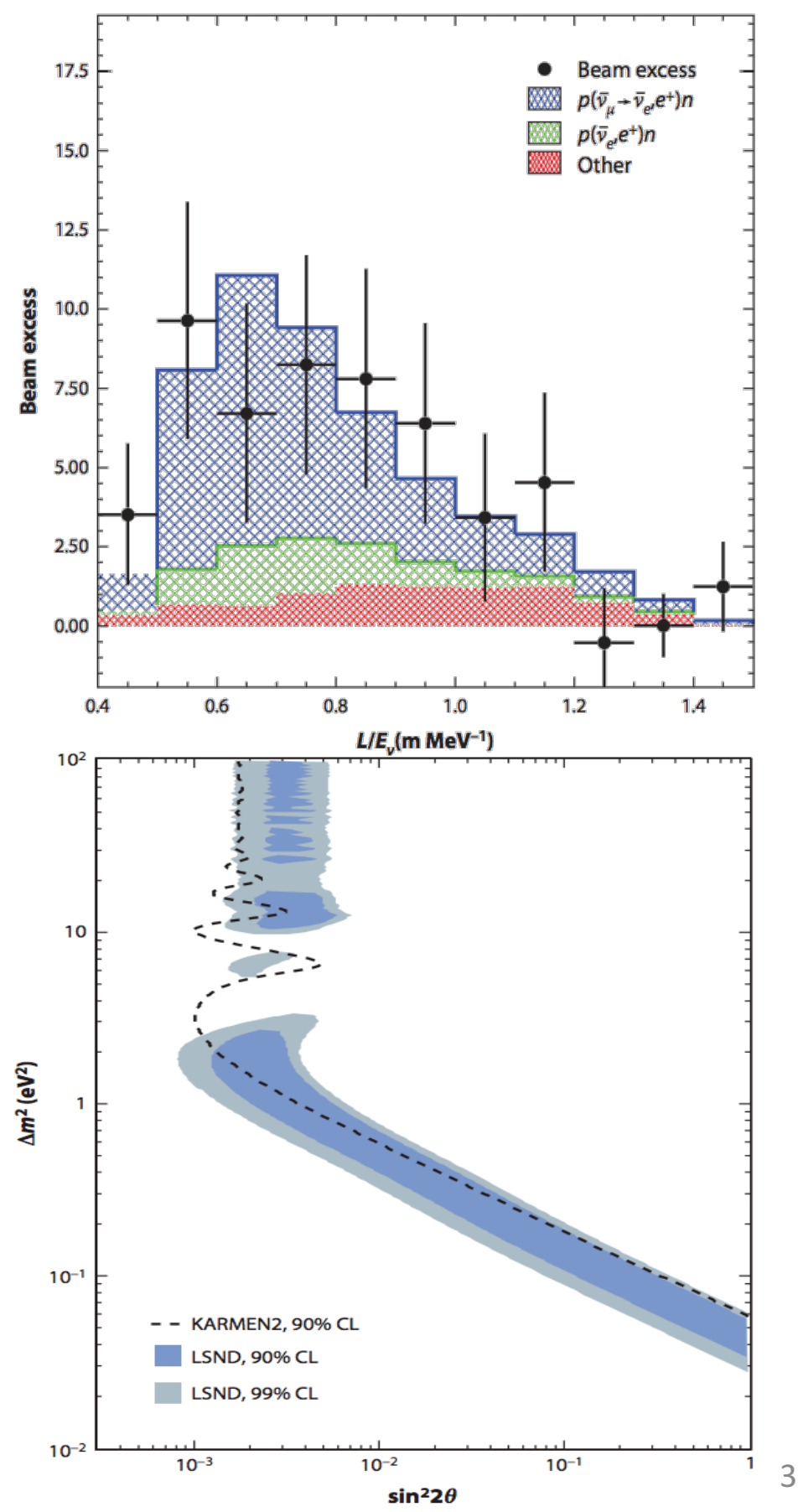

Annu. Rev. Nucl. Part. Sci., 63(1), 45-67. 


\section{MiniBooNE}

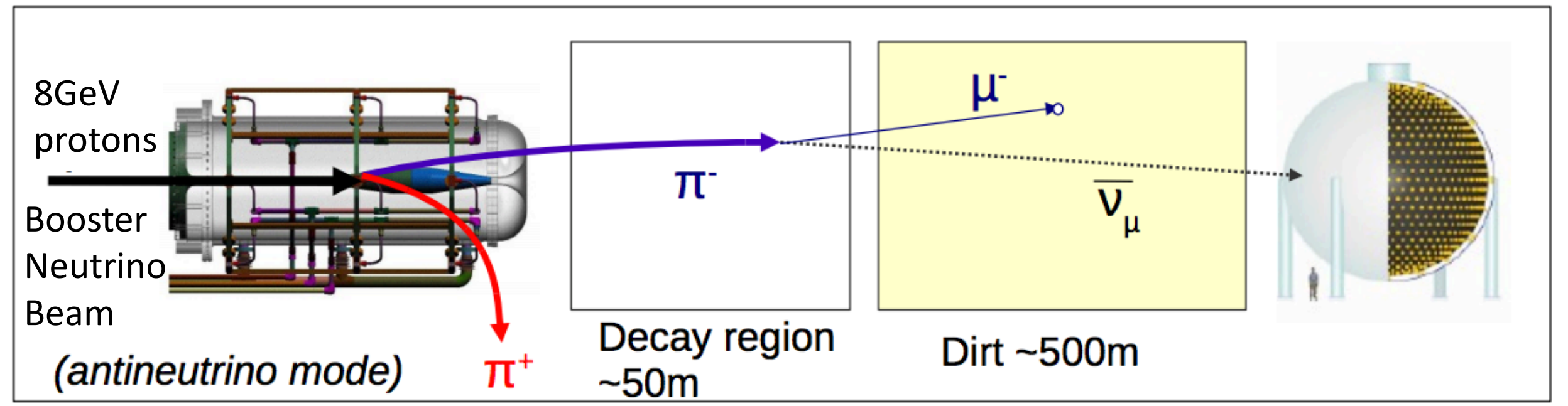

Similar L/E

MiniBooNE 500m/500MeV

- LSND $\sim 30 \mathrm{~m} / 30 \mathrm{MeV}$

800-ton mineral oil Cherenkov detector

Different systematics

- Different flux, event signatures, and backgrounds from LSND

Horn polarity determines $v$ or $\bar{v}$ mode

Flux monitor for short baseline neutrino program (SBN)

Well-understood detector with 26

publications(4900+ citations) in different channels, as well as recent

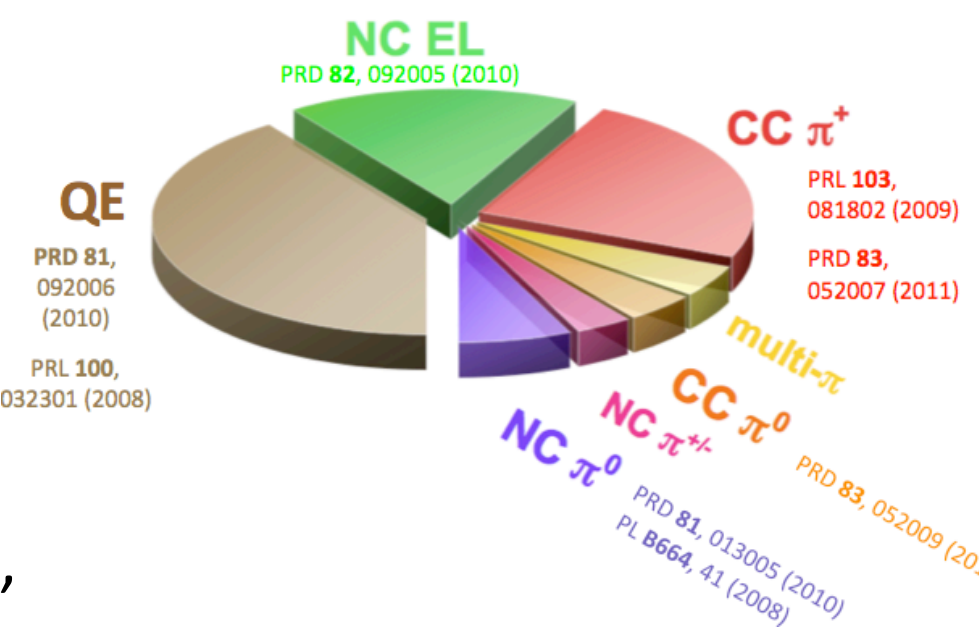

- $v_{\mu}$ from $K^{+}$decay at rest from NuMI beam

- Dark matter search 


\section{KDAR}

KDAR=Kaon Decay At Rest

KDAR neutrinos from the NuMI beamline absorber have been isolated based on energy reconstruction and timing.

- First measurement of $\omega$ (energy transferred to the nucleus) with a known energy, weakinteraction-only nuclear probe.

- Results provide a standard candle for understanding $v_{\mu}$ CC events at a known energy (236 MeV).

An associated data release website allows any model prediction $\left(T_{\mu}\right.$ or $\omega$ ) to be compared with the data.

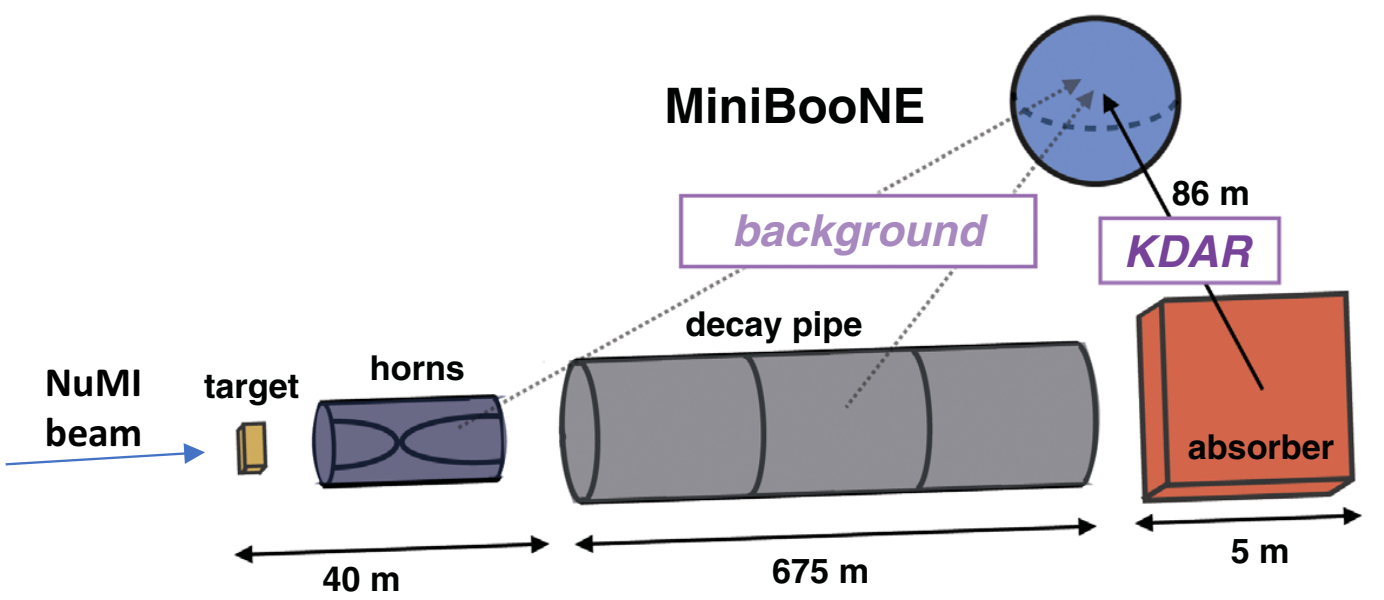

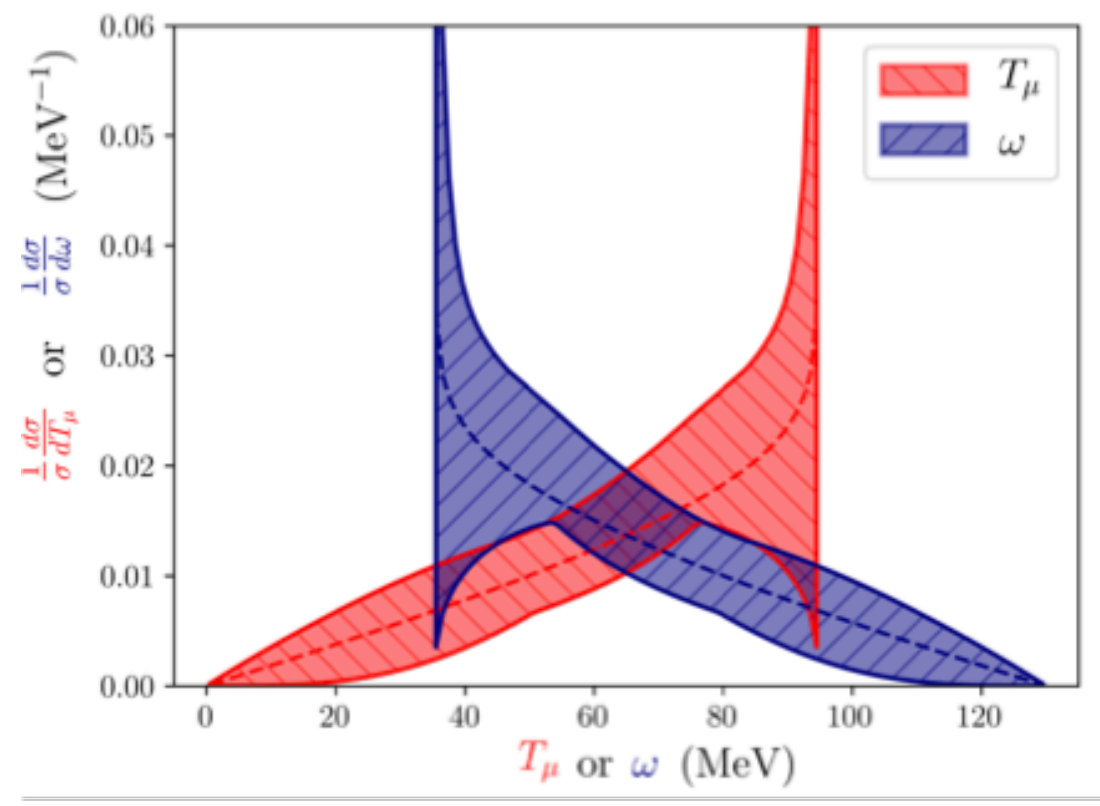

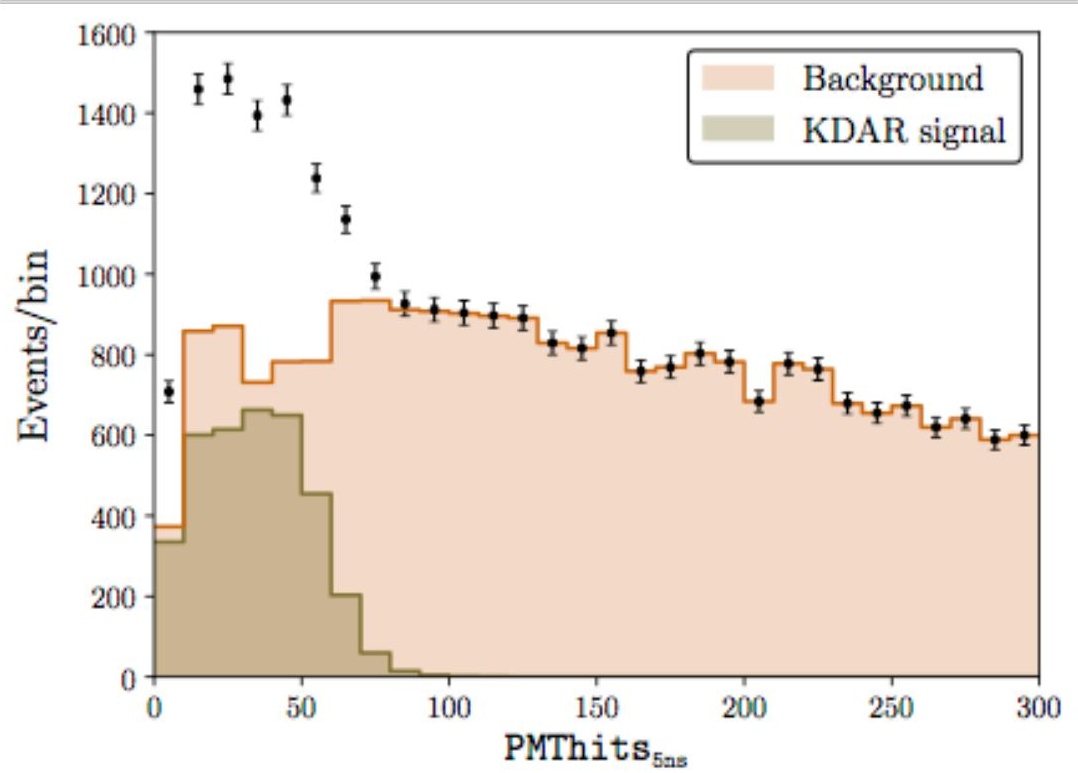




\section{Dark Matter Search in Beam-Dump Mode}

PRL 118, 221803 (2017)

\section{Editor's Suggestion}

First dedicated search for direct detection of accelerator-produced dark matter in a proton beamline

Searched for the dark matter to elastically scatter off nucleons

Beam-dump mode reduced the $v$ flux by $\sim 50$

The goal was to test vector portal model interpretation of g-2 (ruled out)

At time of publication: set world leading limits in the vector portal dark

matter model with a dark matter mass

between 0.01 and $0.3 \mathrm{GeV}$

New results are expected later in 2018

Inelastic scatter to produce $\pi^{0} \mathrm{~s}$ through $\Delta$ decay

Elastic scattering off electrons

Target Decay Pipe

Beam Dump
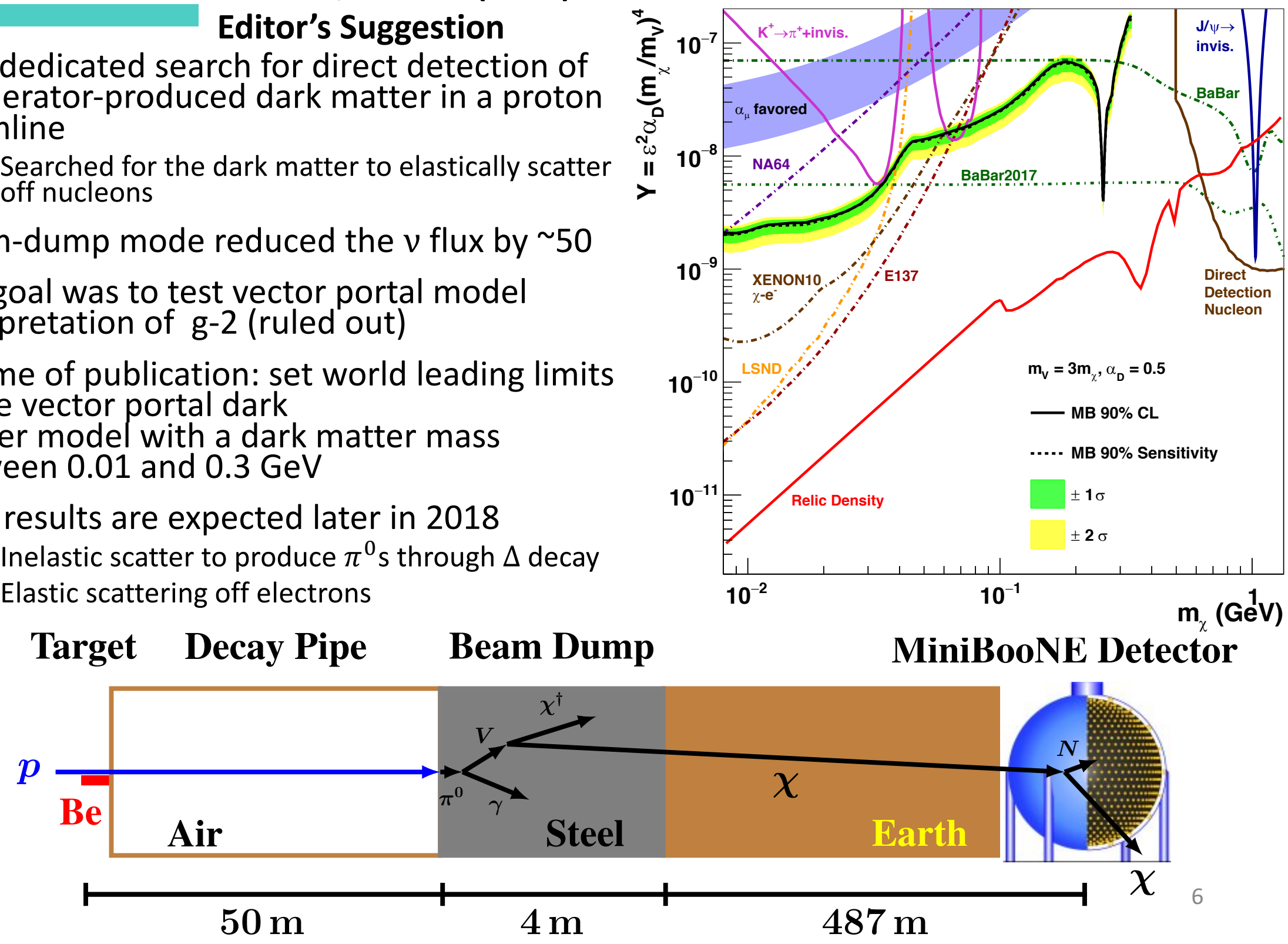


\section{New MiniBooNE Oscillation Results with}

\section{Neutrino Data Set Doubled}

arXiv:1805.12028

submitted to PRL

- Extra data allows better calibrations and cross checks

- Second data set to look at consistency

- Improved background estimates from observed data and constraints

- Dirt and $\pi^{0}$

- Larger data set leads to smaller statistical uncertainty on signal and background measurements 


\section{Data Set}

- $15+$ years of running in neytrino, antineutrino, and beam dump mode. More than $30 \times 10^{20}$ POT to date.

- Result of a combined $12.84 \times 10^{20}$ POT in $v$ mode $+11.27 \times$ $10^{20}$ POT in $\bar{v}$ mode is presented in this talk

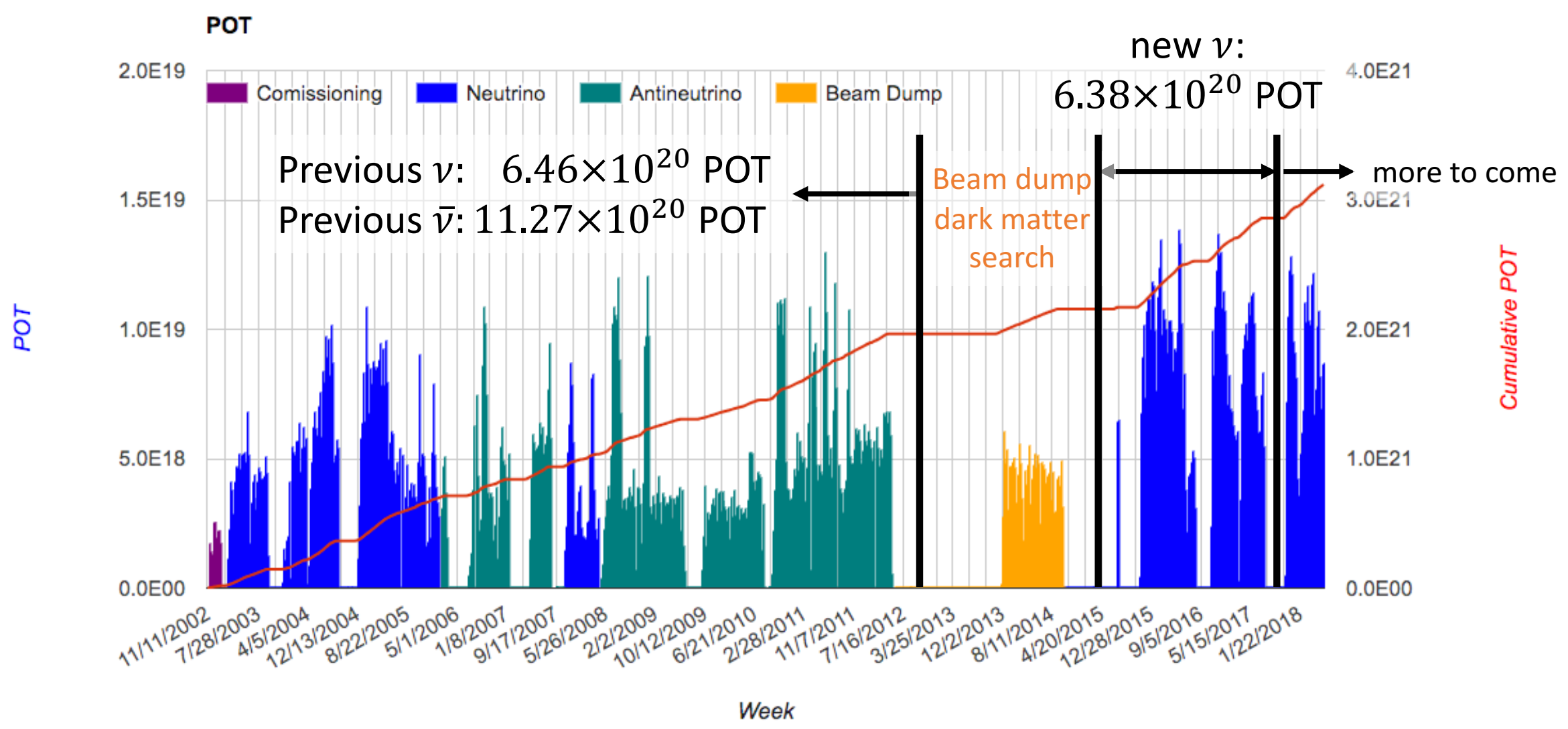




\section{Event Signatures}

- Examples of $v_{\mu}$ CCQE $v_{e} \mathrm{CCQE}$, and $\mathrm{NC} \pi^{0}$ event topologies

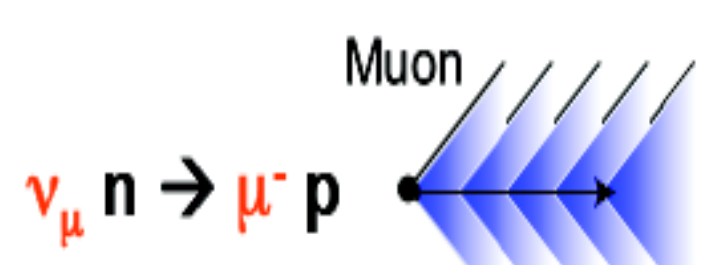

- Use primarily Cherenkov light

- Compare fits of different track reconstruction hypotheses for PID

- Insensitive to the difference between single photon and single electron (time of flight might help)

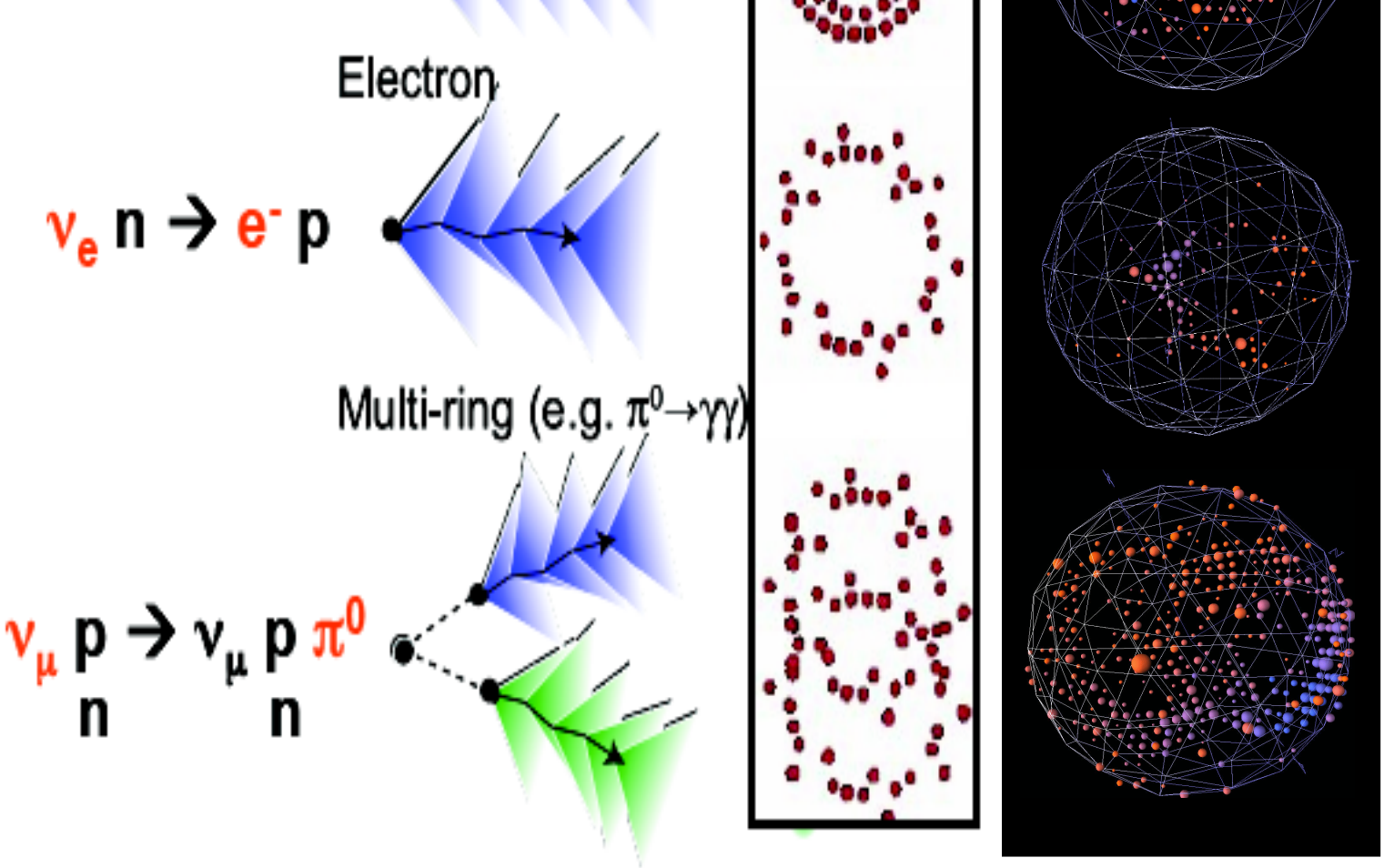




\section{Data vs MC (Selection Process)}

$m_{\gamma \gamma}$ is shown

Cuts are applied in the order of

a. Only precuts (no PID cut)

b. e- $\mu$ Likelihood cut

c. e- $\pi$ Likelihood cut

d. $m_{\gamma \gamma}$ cut

- Background outside the oscillation cut window is well understood by MC Other two PID distributions are in the backup slides
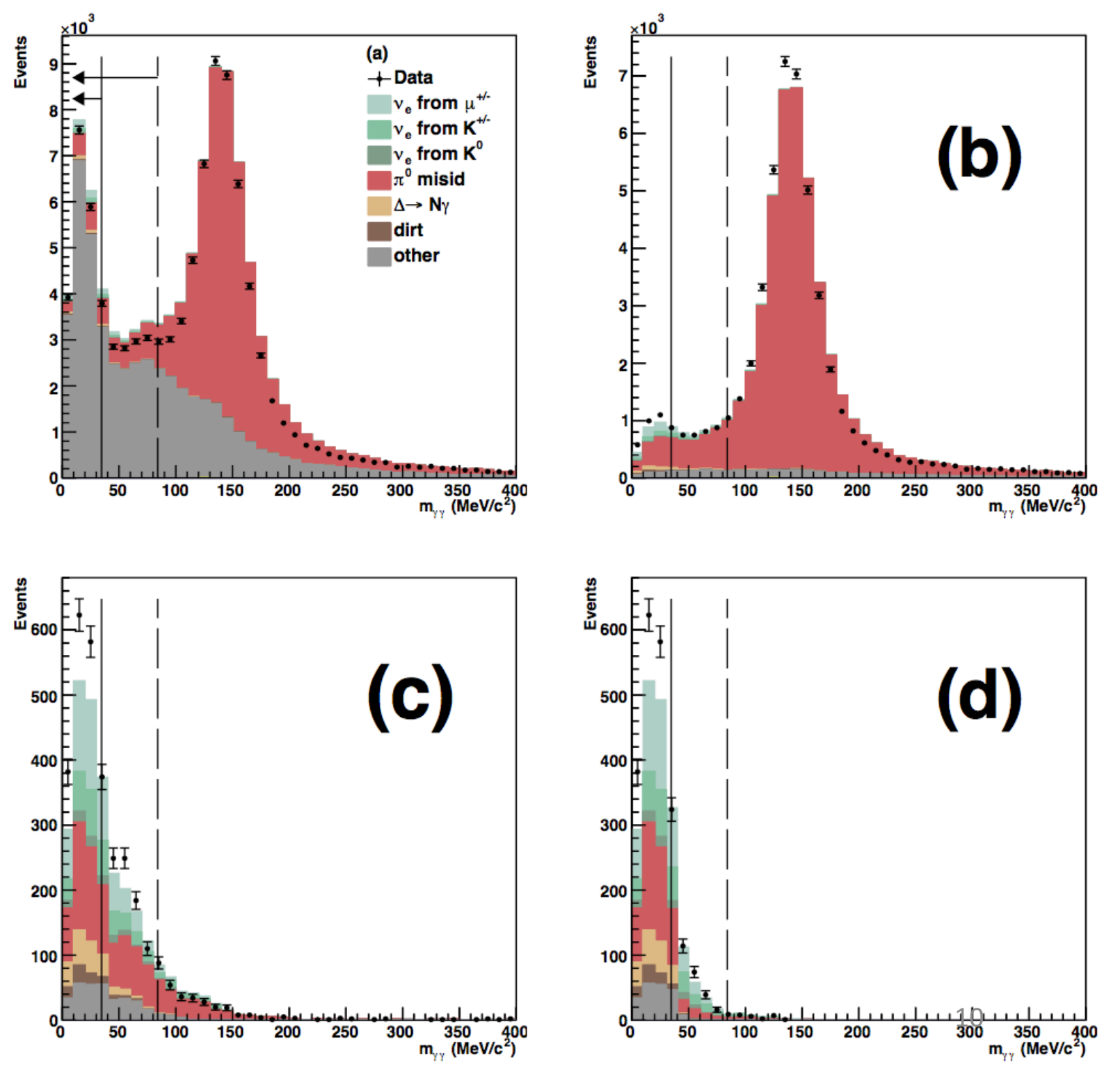


\section{MiniBooNE Analysis}

- Standard 2-v oscillation model is used:

$$
P(L, E)=\sin ^{2} 2 \theta \times \sin ^{2}\left(1.267 \Delta m^{2} L / E\right)
$$

- $\sin ^{2} 2 \theta \equiv \sin ^{2} 2 \theta_{\mu e} \approx 4\left|U_{\mu 4}\right|^{2}\left|U_{e 4}\right|^{2}$ at the MiniBooNE mass splitting range

- In a 3 (active) +1 (sterile) model, $\sin ^{2} 2 \theta_{\mu e}$ is assumed small

- $v_{e}$ CCQE-like events are constrained by the $v_{\mu}$ CCQE-like events

- Maximum likelihood is used

- For a $v+\bar{v}$ analysis, a simultaneous fit was conducted for $v_{e}, v_{\mu}, \bar{v}_{e}$, and $\bar{v}_{\mu}$ distributions 


\section{Excess}

\begin{tabular}{|c|c|c|c|}
\hline & $\begin{array}{c}v \text { mode } \\
12.84 \times 10^{20} \text { POT }\end{array}$ & $\begin{array}{c}\bar{v} \text { mode } \\
11.27 \times 10^{20} \text { POT }\end{array}$ & Combined \\
\hline Data & 1959 & 478 & 2437 \\
\hline $\begin{array}{l}\text { Unconstrained } \\
\text { Background }\end{array}$ & 1590.5 & 398.2 & 1988.7 \\
\hline $\begin{array}{l}\text { Constrained } \\
\text { Background }\end{array}$ & 1577.8 & 398.7 & 1976.5 \\
\hline Excess & $\begin{array}{c}381.2 \pm 85.2 \\
4.5 \sigma\end{array}$ & $\begin{array}{c}79.3 \pm 28.6 \\
2.8 \sigma\end{array}$ & $\begin{array}{c}460.5 \pm 95.8 \\
4.8 \sigma\end{array}$ \\
\hline $\begin{array}{l}0.26 \% \text { (LSND) } \\
v_{\mu} \rightarrow v_{e}\end{array}$ & 463.1 & 100.0 & 563.1 \\
\hline
\end{tabular}

- Total excess for neutrino + antineutrino: $460.5 \pm 95.8(4.8 \sigma)$

- Combined with LSND $(3.8 \sigma)$, total significance is at $6.1 \sigma$ 


\section{Excess: Old vs New in $\boldsymbol{v}$ Mode}
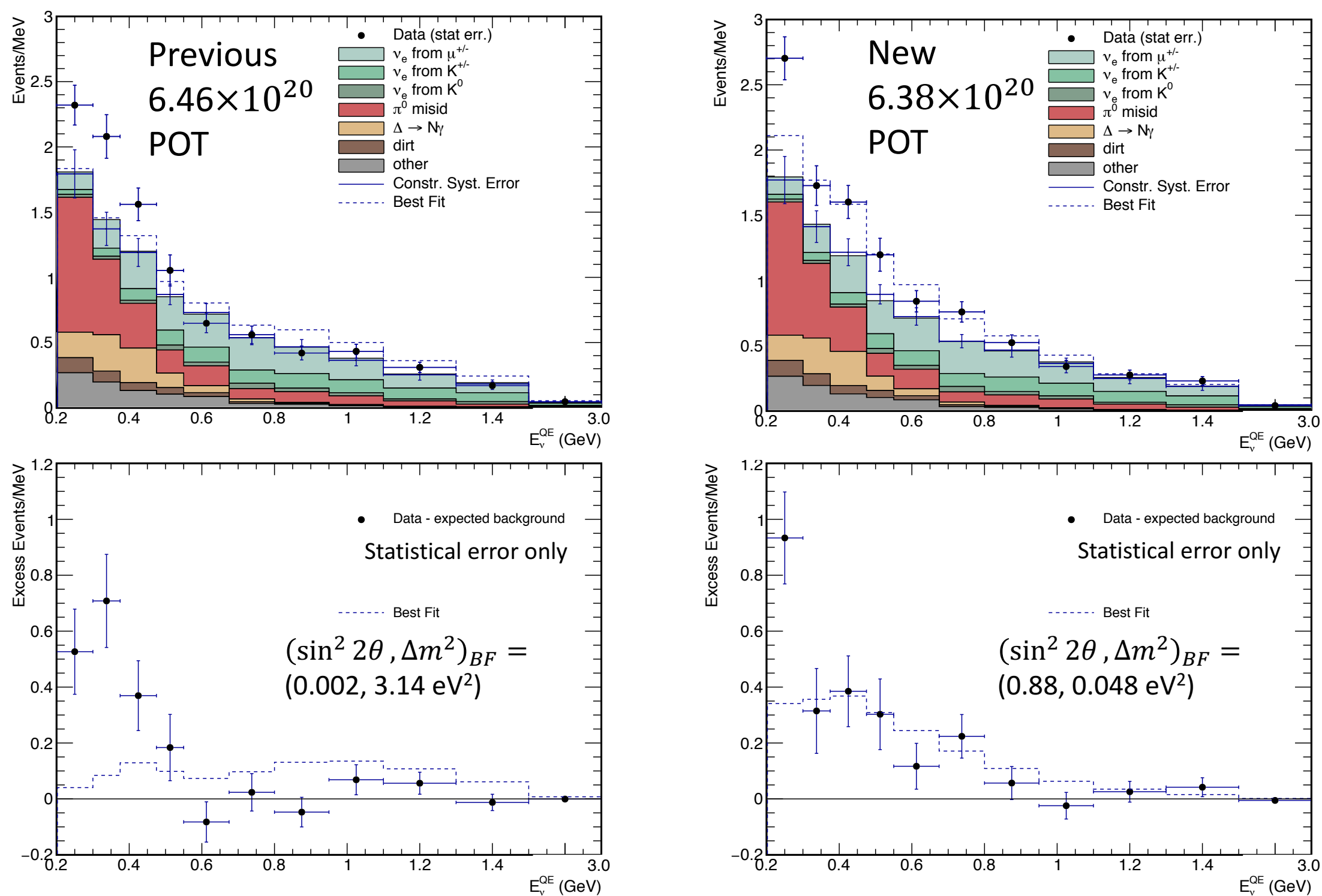

The observed $v_{e}$ spectra are statistically consistent between the new and previous data sets (KS prob $=76 \%$ ) 


\section{Excess: Neutrino vs Anti-neutrino}

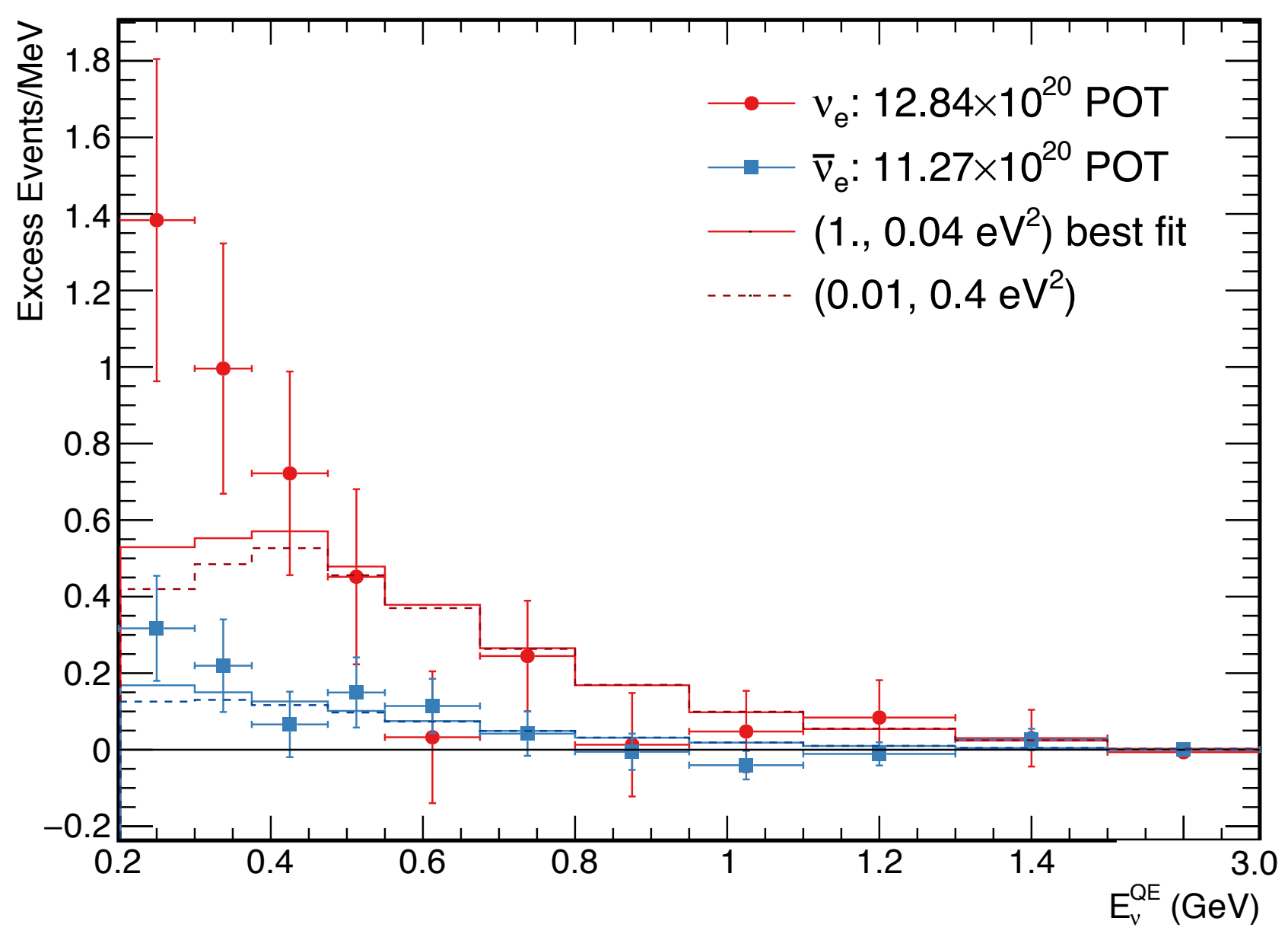

- Excess in neutrino and antineutrino mode is qualitatively consistent 


\section{Allowed Region}
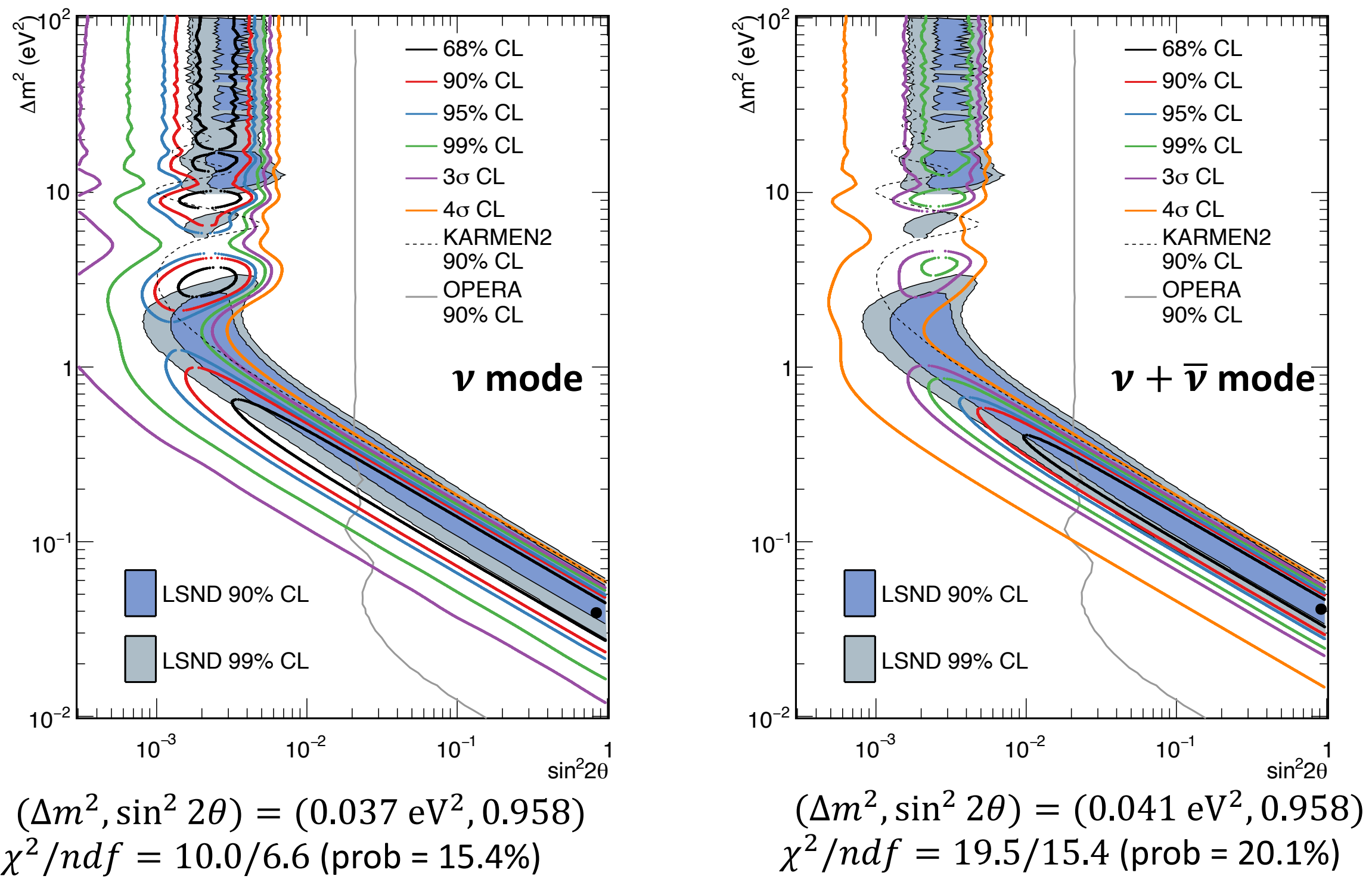

$\left(\Delta m^{2}, \sin ^{2} 2 \theta\right)=\left(0.037 \mathrm{eV}^{2}, 0.958\right)$ $\chi^{2} / n d f=10.0 / 6.6($ prob $=15.4 \%)$ 


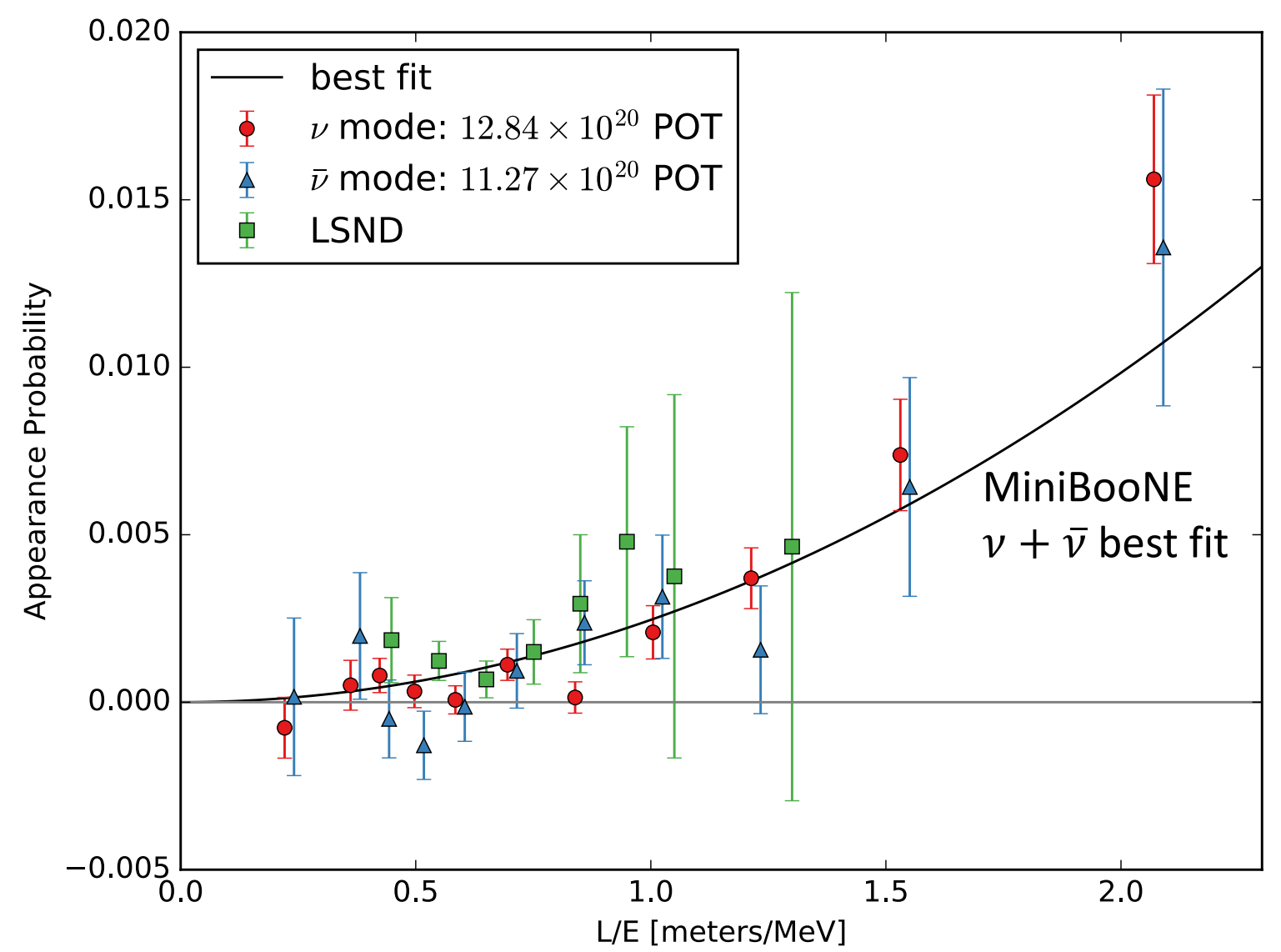

- Average $E_{v}^{Q E}$ of each bin is used

- MiniBooNE neutrino, MiniBooNE antineutrino and LSND are consistent in appearance probability and L/E 


\section{Combined Fit with LSND}

- Combined fit of

MiniBooNE $v+\bar{v}$

mode and LSND is at

$6 \sigma$ level

Assuming no correlation between MiniBooNE and LSND

- Best fit of MiniBooNE and LSND combined is consistent with our latest result

- Note: a large $\sin ^{2} 2 \theta$ is unphysical for a pure 3+1 model

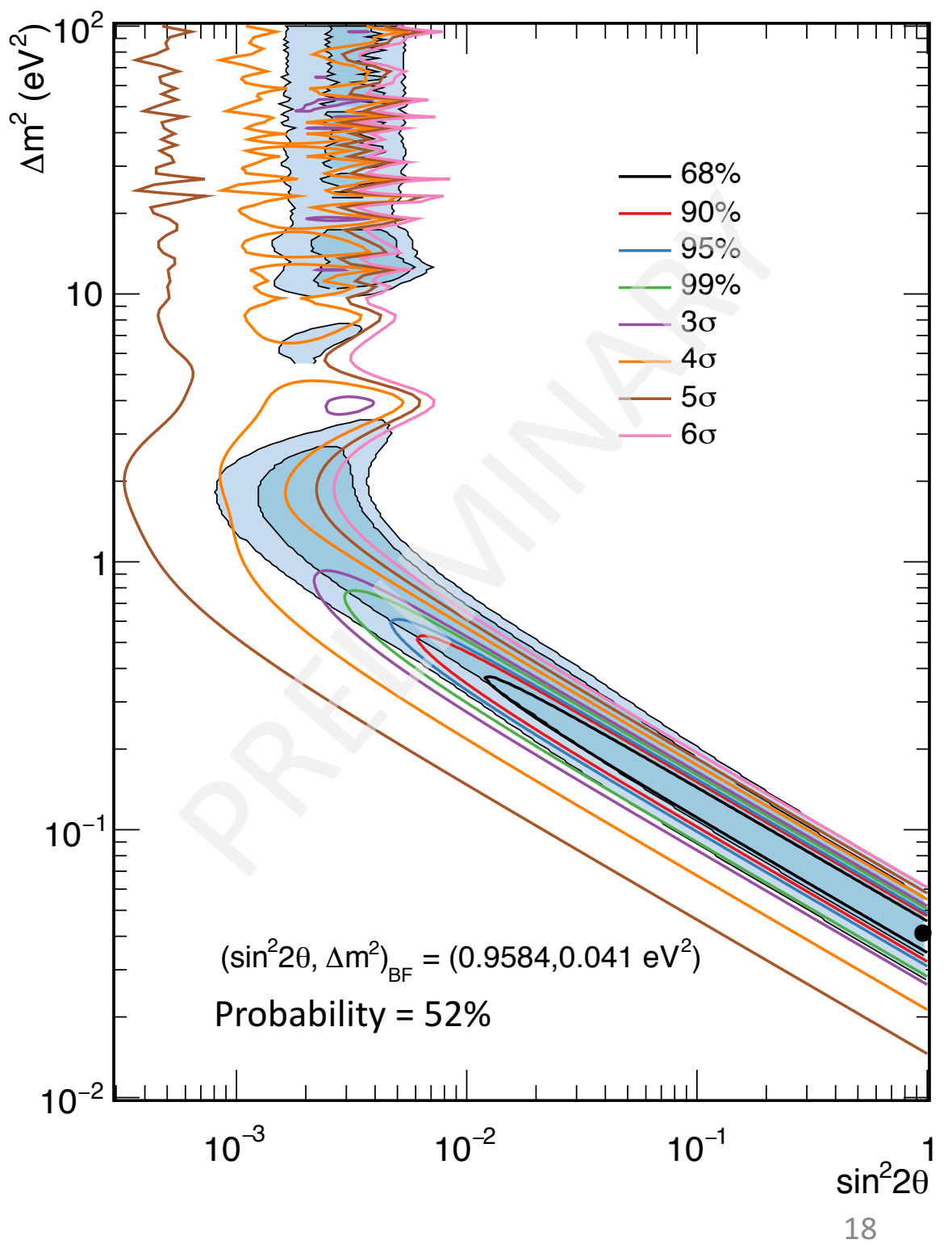




\section{Example of an Empirical Exotic Model: An MSW-Like Resonance}
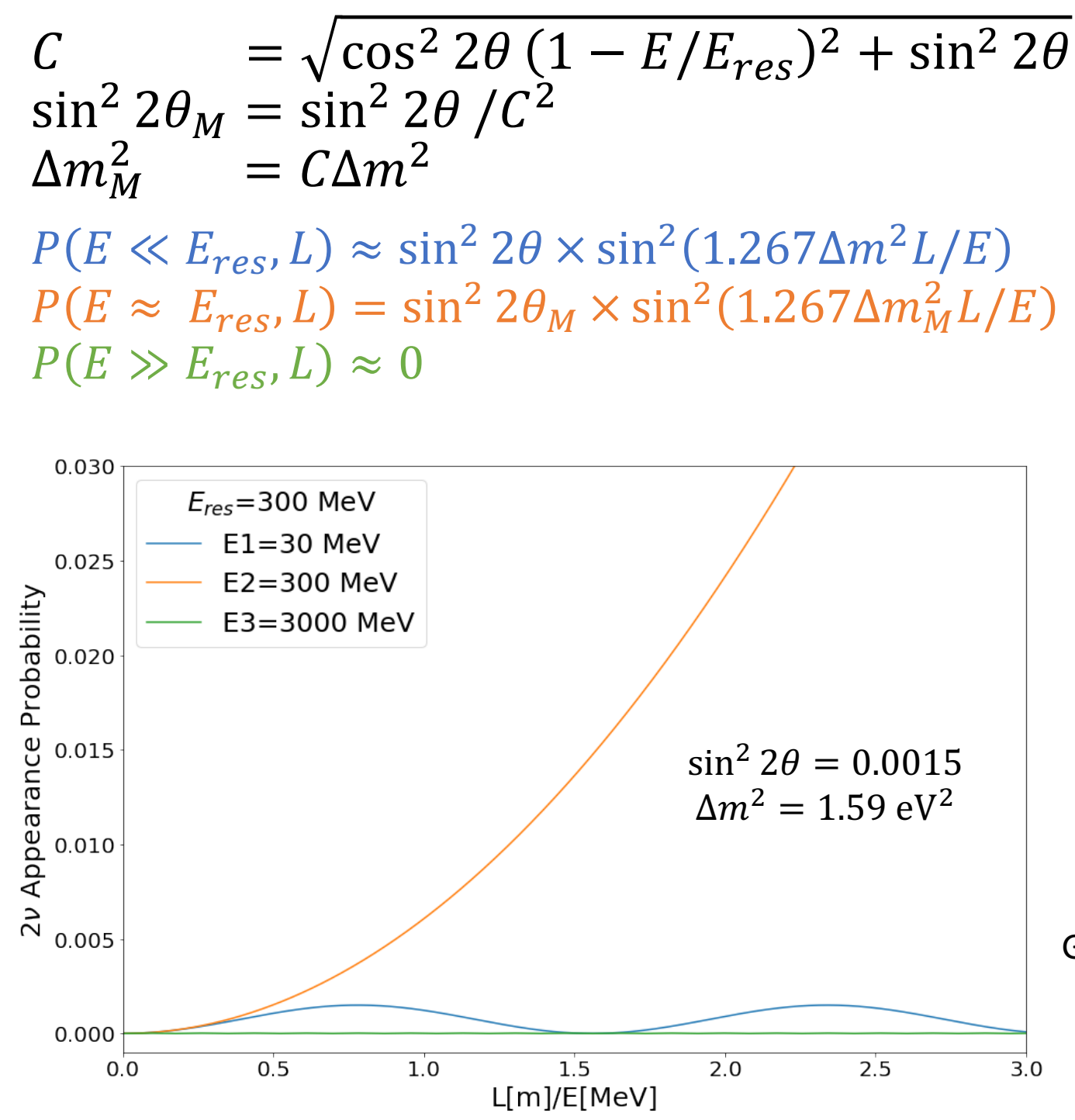

Insipred by J. Assadi et al., arXiv:1712.08019 \& G. Karagiorgi, M. H. Shaevitz, J. M. Conrad arXiv:1202.1024 


\section{Conclusion}

- MiniBooNE confirms LSND excess at $4.8 \sigma$, with a combined significance at $6.1 \sigma$

- MiniBooNE continues data-taking, and analysis in the future will include time-of-flight information to better constrain backgrounds

- MicroBooNE will confirm whether excess is due to electrons or photons

- SBN will confirm whether the excess is due to neutrino oscillations

Thanks to Fermilab for MiniBooNE operation (15 y) $\&$ for great beam delivery 